\title{
Students Performance Evaluation using MCDM Methods through Customized Software
}

\author{
Pavani Sirigiri \\ Dr. C. V. Raman University, \\ Bilaspur, CG
}

\author{
H.S. Hota \\ Guru Ghasidas University \\ Bilaspur, CG
}

\author{
L.K. Sharma \\ National Institute of Occupational \\ Health, Ahmedabad, GJ
}

\begin{abstract}
Multi criterion Decision Making (MCDM) methods are widely applied in many domains for finding rank based on the available conflicting nature of the criteria and to choose the best alternative. MCDM methods comprise of complex mathematical calculations which required time to solve, especially, when number of criteria and alternatives are more. In order to automate the process software is required to develop, which will provide an interactive and dynamic way to solve problems with $\mathrm{n}$ numbers of ranks of the alternatives. This paper introduces developed software for solving MCDM based problems, including many MCDM methods and to evaluate the performance of software with two different case studies related to the performance of students.
\end{abstract}

\section{General Terms}

Decision Support System, Fuzzy, Soft computing.

\section{Keywords}

Student Performance Ranking, Multi Criterion Decision Making, Fuzzy TOPSIS, Fuzzy.

\section{INTRODUCTION}

In current competitive educational environment, a continuous evaluation system is being adopted and performance of students [13] [14] must be checked continuously. Students' performance may be verified in many ways which is sometimes difficult due to many conflicting criteria [1] [2] [3] [4], a human expert can't do the same, but are necessary to determine to identify strengths, and to identify areas of performance that may need special attention and remediation. MCDM methods [5] [6] [7] may be better alternatives for solving these types of problems and are gaining importance as potential tools for analyzing complex real problems due to their inherent ability to judge different alternatives (choice, strategy, policy, scenario can also be used synonymously) on various criteria for possible selection of the best/suitable alternatives. These alternatives may be further explored indepth for their final implementation. These analysis have some unique characteristics such as the presence of multiple non-commensurable and conflicting criteria [8][9][10], different units of measurement among the criteria, and the presence of quite different alternatives.

In this paper, user-friendly developed software for solving complex decision making problems using MCDM methods [17] in an interactive way is introduced. This software provides many options to select number of criteria, alternative and MCDM methods. The proposed software is tested with two students' performance related problems and the obtained results are analyzed.

\section{RELATED WORK}

Many literatures are available for applying different domains of MCDM methods. Cebeci [12] used fuzzy AHP-based decision support system for selection Enterprise Resource
Planning (ERP) systems in the textile industry by using a balanced scorecard. Dagdeviren et al. [9] proposed the weapon selection using AHP and TOPSIS method under fuzzy environment. The AHP was used to analyze the structure of the project selection and Fuzzy TOPSIS method was used to obtain final ranking. Nepal et al. [3] presented a fuzzy-AHP framework to determine the prioritization weights of customer satisfaction attributes to facilitate the target planning decision in order to improve vehicle design. Amiri [22] proposed the evaluation and selection of project for oil field development by using the AHP and the fuzzy TOPSIS methods. In this study, methodology was tested on a real world example for both the conventional AHP and fuzzy AHP approaches for CMMS selection. Duran [10] implemented fuzzy-based AHP methodology for comparative evaluation of a number of computerized maintenance management system (CMMS). Kutlu and Ekmekcioglu [2] proposed fuzzy TOPSIS based scoring off for failure mode and effects analysis, which are ranked to prioritize the failure modes and evaluate the risk factors of each potential failure mode in linguistic variables. Buyukozkan and Cifci [7] proposed a combined multiple criteria decision making methodology containing fuzzy AHP and fuzzy TOPSIS to evaluate a set of hospital web sites, alternatives in order to reach to the best qualified alternatives that satisfies the needs and the expectations of customers. Chou et al. [14] implemented a combination of fuzzy analytic hierarchy process and fuzzy decision making trial and evaluation laboratory method in human resource for science and technology (HRST). The result of AHP was used for outreach personnel to improved performance in a timely basis for HRST. Awasthi and Chauhan [1] proposed approach to selecting sustainable city logistics initiatives for cities.

MCDM methods are recently developed and applying in the evaluations of teacher-student performance, ranking for teachers, etc. The main goal of these methods is to decide the ranking among the various available alternatives where the human intelligence is very difficult to apply due to the conflicting nature of the criteria. Sen and Cinar [4] evaluated and pre-allocated of the operator with multiple skills using integrates a fuzzy AHP method. The first ten students' feedback opinion of particular department has been considered to evaluate teacher performance in this study. Ghosh [6] utilized AHP and TOPSIS methods for evaluation of the best faculty members. Mehregan et al. [21] introduced an approach to e-learning system assessment by identifying and prioritizing the preliminary e-learning critical success factors using fuzzy AHP in ranking modern education systems. Chrysafiadi and Virvou [8] proposed evaluation of the integration of a fuzzy logic technique into the student model of a web-based educational environment for teaching the programming language. Gurupur et al. [13] proposed a tool using concept maps and Markov chains in evaluating a student's understanding of a particular topic of study using concept maps. Jeremic et al. [15] presented evaluation of a 
student model Design Pattern Teaching Help System (DEPTHS), an intelligent tutoring system for learning software design patterns.

\section{MCDM BASED CYSTOMIZED SOFTWARE}

Automation of any complex mathematical problem is always needed to speed up the problem solving process and to analyze the results in a comparative manner. MCDM methods consist complex mathematical calculation and it became tedious when number of criteria and alternative are more, in this situation a customized software may be very much helpful.

Among many MCDM methods, the analytic hierarchy process (AHP), as proposed by Saaty [11] becomes popular. Recently modification to the AHP is considered to be more consistent than the original approach. AHP is an approach to decision making that involves structuring multiple choice criteria into a hierarchy, assessing the relative importance of these criteria, comparing alternatives for each criterion, and determining an overall ranking of the alternatives. The output of the AHP is prioritized ranking indicating the overall preference for each of the decision alternatives eventually help the decision maker to select the best approach. The technique for order preference by similarity to ideal solution (TOPSIS) was used in many multi criteria applications [1][2][6][7][9][19]. The basic concept of this method is that the selected alternative should have the shortest distance to the positive ideal solution and the farthest distance from the negative ideal solution [17]. The TOPSIS method assumes that each criterion tends toward a monotonically increasing or decreasing utility [18]. Therefore, it is easy to define the positive and negative ideal solutions. The Euclidean distance approach was proposed to evaluate the relative closeness of the alternatives to the ideal solution. Thus, the preference order of the alternatives can be derived from a series of comparisons of these relative distances. The Positive ideal Solution maximizes the benefit criteria and minimizes the cost criteria, whereas the Negative ideal solution maximizes the cost criteria and minimizes the benefit criteria. Fuzzy [16] versions of these MCDM methods are the fuzzy AHP and the fuzzy TOPSIS methods which can be used when the decision making problems are fuzzy in nature instead of crisp. Fuzzy logic can be used to deal with this type of problem. The FAHP method is an advanced analytical method which is developed from the AHP and this method is often criticized for its inability to adequately handle the inherent uncertainty and imprecision associated with the mapping of the decision-makers perception of exact numbers. The fuzzy TOPSIS approach enables experts the fuzzy TOPSIS method proposed by Yong [19] to evaluate web services for selection. TOPSIS uses triangular fuzzy numbers representing linguistic variables as the weights of criteria and as the ratings of web services which can be transformed into crisp numbers. The transformation is performed by the graded mean integration, representation method proposed by Chou [20]. The canonical representation of the addition and the multiplication operations on triangular fuzzy numbers is then used to obtain the PIS and the NIS. To avoid the problem with doubling weightings on each alternative, the distance of each alternative web service from the PIS and the NIS is measured by Minkowski distance function. As a result, the preference order of available alternative web services can be identified accordingly. Based on the graded mean integration, representation of triangular fuzzy numbers and the canonical representation of addition and multiplication of triangular fuzzy numbers, the procedure of the fuzzy TOPSIS method can be performed in an efficient way. This property can reduce the computational complexity in the decision making process.

The customized software is developed by using .NET programming language as front end and MS-Access and flat file as back end. The developed software provides interactive Graphical User Interface (GUI). Various components of the software and its utilization of evaluation of performance of students are explained in subsections.

\subsection{User-Login Screen}

A user login screen of the software can be seen in Fig. 1 through which a valid user can enter his/her user Id and password to utilize the software.

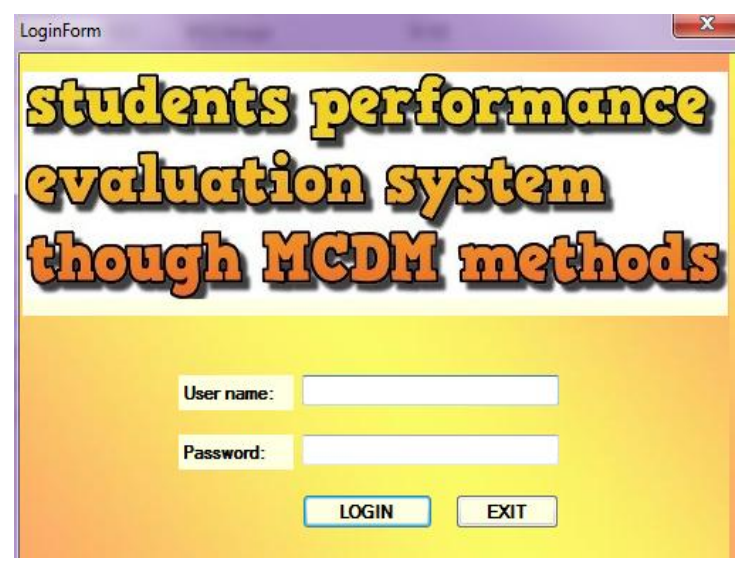

Fig 1: A user Login screen of customized software

\subsection{Selection of MCDM methods}

Software can solve decision making problem using many MCDM methods and user can choose either one method or many other methods to compare the results. Before starting the evaluation process by any of the MCDM methods, a number of criteria and a number of alternatives are to be decided. There are two ways for selecting criteria and alternatives: First is to directly input number of alternative and criteria and second is to load the input file consisting number of criteria and alternative along with data. The entire process of calculation of MCDM methods may be stored in file by providing file name in test name text box as shown in Fig. 2.

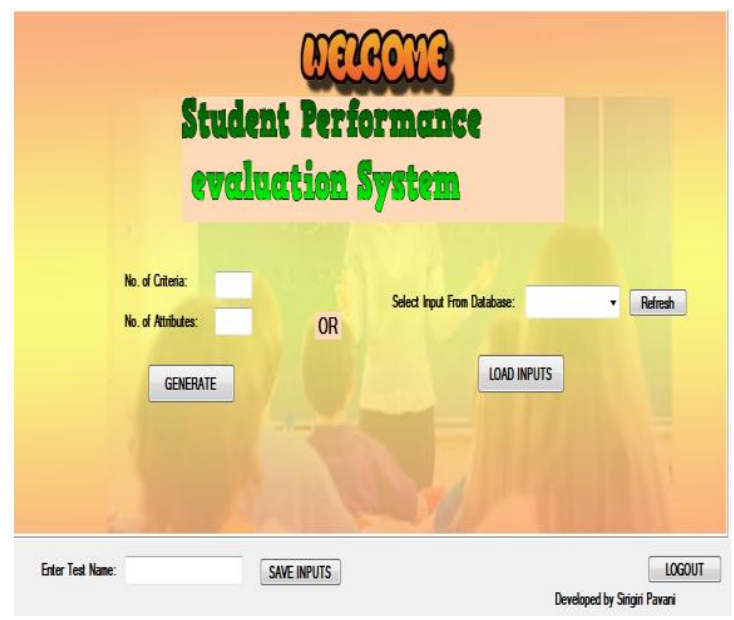

Fig 2: An Interface to provide criteria and alternative 


\section{PERFORMANCE EVALUATION OF STUDENTS}

For experimental work and to evaluate the performance of developed software, two cases to evaluate Students' performance are considered: (1) selection of the best project and (2) evaluate students' performance on utilization of Information Communication Technology (ICT) in teachinglearning process. The overall process is shown in Fig. 3 in which 5 criteria are decided for each decision making problem based on the opinion of experts in this domain and alternatives are obviously students. Two different case studies mentioned as above are explained in the following sections in detail.

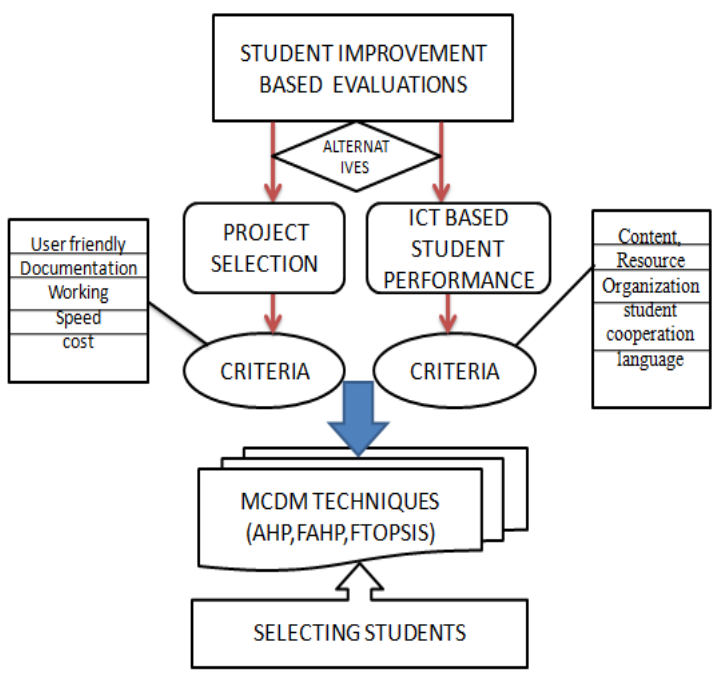

Fig 3: Hierarchical structure for applying MCDM methods for evaluation of student performance

\subsection{Case 1: Student Project Evaluation}

Student's projects provide illustrative examples of the issues affecting student perceptions and expectations regarding quality and standards for the subject. All students who are in the information technology based program in higher learning institutions. Every student project needs to be carefully evaluated by a supervisor and a group of panels as to ensure that they are fairly rated and be accorded standardized marks. The evaluation of the project is a multi-criteria decision problem, a few of which are fuzzy in nature. The fuzziness of criteria makes the evaluation process very intricate and complicated. This paper proposes a project evaluation framework for the integration of Weighted Model, fuzzy multi criteria decision making approach based on Fuzzy AHP and Fuzzy TOPSIS as a decision support system. Criteria weighting is determined based on weighted model and fuzzy AHP. Fuzzy TOPSIS approach is used to rank the project. Finally, a set of numerical data is used to illustrate the procedure of the methods and then the ranking of the project is produced based on the experts' evaluation. The application is conducted to illustrate the utilization of the model for the project evaluation problem. Additionally, in the application, it is shown that the calculation of the criteria weights is important in both methods and they could change the ranking. The decision maker can use these different weight combinations during the decision making process based on the priority.

Once the data related to project evaluation of students are uploaded in software. The software automatically calculates all the intermediate results and finally display rank as shown in Fig. 4. For the simplicity six alternatives are considered, however, a number of alternatives may be selected as per requirement.

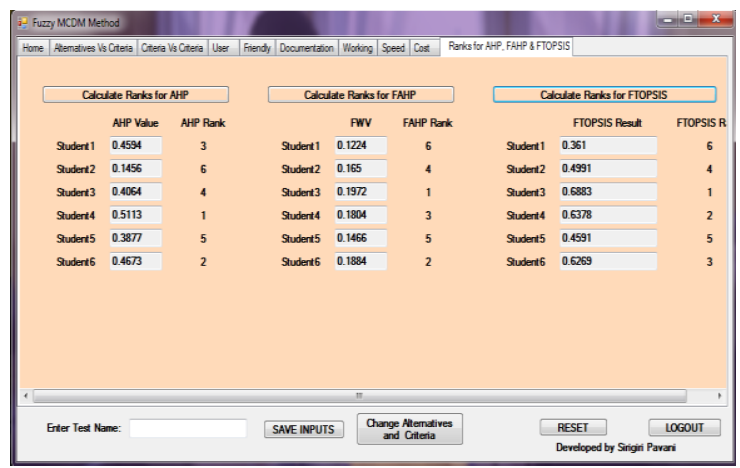

Fig 4: Rank obtained through software using AHP, FAHP and FTOPSIS for student project evaluation

The above figure shows a comparative ranking of the alternatives using AHP, FAHP and FTOPSIS, as it is clear the rank of the different alternatives are different in case of different MCDM methods. If we consider first alternative (Student 1) the rank is 3,6 and 6 respectively in case of AHP, FAHP and FTOPSIS, which is quite obvious. Before obtaining rank consistency is also checked and found under the limit which proves that the assigned weights are appropriate.

\subsection{Case 2: Students' Performance Evaluation on Utilization of ICT}

The use of information and communication technology (ICT) such as Internet applications, CD-ROMs, video technology and various computer attachments and software programs have caused many changes in society. ICT has introduced numerous trends in different areas of human life with the integration of the education field. Now day's students are mostly using the internets. E-learning (EL) is the use of Information and Communication Technology e.g. Internet, Computer, Mobile phone. In order to verify the robustness of software one more case study is considered as students' performance evaluation on utilization of ICT with six criteria as explained above and with six alternatives. Once again data are uploaded in the software and rank of the alternatives are calculated using the same three MCDM methods and obtained ranks are displayed in the software itself as shown in Fig. 5.

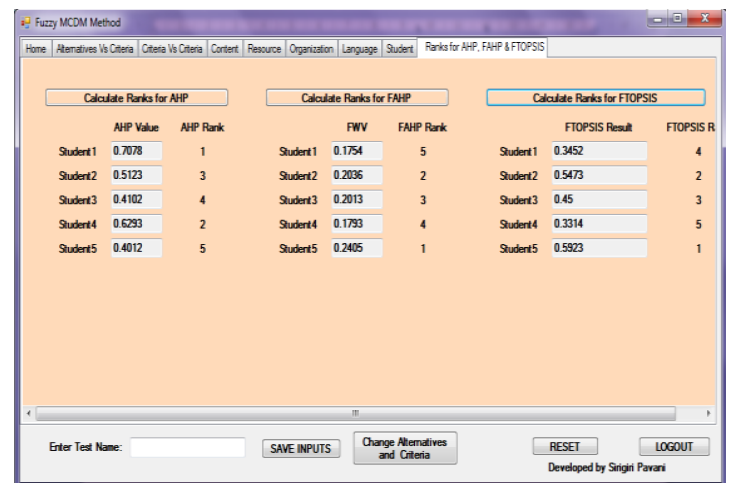

Fig. 5: Rank obtained through software using AHP, FAHP and FTOPSIS for students' performance evaluation on utilization of ICT

In order to start the process, an expert assigned weights each criterion are uploaded into the software to calculate the 
consistency ration (CR) and then consistency Index (CI) and are found satisfactory. As similar to case 1, here also AHP method student 1 is getting the rank-1 and FAHP and FTOPSIS method student 5 is getting rank 1 .

\section{CONCLUSION}

For complex decision making problems, MCDM methods are widely used in various domains. Evaluation of students' performance based on various conflicting criteria may also be treated as one of the complex decision making optimization problems. The customized software may help to do the experimental work and to compare the results. Two different case studies related to evaluation of performance of students are considered. The software provides an interactive way to choose the number of criteria and alternatives and to compare the results.

\section{REFERENCES}

[1] Awasthi, A. and Chauhan, S.S. 2012. A hybrid approach integrating affinity diagram, AHP and fuzzy TOPSIS for sustainable city logistics planning, Applied Mathematical Modelling, 36(2), 573-584.

[2] Kutlu, AC and Ekmekcioglu, M. 2012. Fuzzy failure modes and effect analysis by using fuzzy TOPSIS-based fuzzy AHP, Expert system with Applications, 39(1), 6167.

[3] Nepal, B., Yadav, O. P. and Murat, A. 2010. A fuzzyAHP approach to prioritized of CS attributes in target planning for automotive product development, Expert System with Applications, 37(10), 6775-6786.

[4] Sen, C. G. and Cinar, G. 2010. Evaluation and preallocation of operators with multiple skills: A combined fuzzy AHP and max-min approach, Expert System with Applications, 37(3), 2043-2053.

[5] Chang, D. Y. 1992. Extent Analysis and Synthetic Decision, Optimization Techniques and Applications, World Scientific, Vol. 1, 352-355.

[6] Ghosh, D. N. 2011. Analytic hierarchy process and TOPSIS method evaluate faculty performance in engineering education, UNIASCIT, 1(2), 63-70.

[7] Buyukozkan, G. and Cifci, G. 2012. A combined fuzzy AHP and fuzzy TOPSIS based strategic analysis of electronic service quality in healthcare industry, Expert System with Applications, 39(3), 2341-2354.

[8] Chrysafiadi, K. and Virvou, M. 2012. Evaluating the integration of fuzzy logic into the student model of a web-based learning environment, Expert System with Applications, 39(18), 13127-13134.

[9] Dagdeviren, M., Yavuz, S. and Kilinc, N. 2009. Weapon selecting using the AHP and TOPSIS methods under fuzzy environment, An Expert system with applications, 36(4), 8143-8151.
[10] Duran, O. 2011. Computer-aided maintenance management system selection based on a fuzzy AHP approach, Advance in Engineering software, 42(10), 821-829.

[11] Saaty, T. L. 1980. The Analytic Hierarchy Process, Planning, Priority Setting, Resource Allocation, McGraw-Hill, New York.

[12] Cebeci, U. 2009. Fuzzy AHP-based decision support system for selecting ERP system in textile industry by using balanced scorecard, Expert System with Applications, 36(5), 8900-8909.

[13] Gurupur, V. P., Jain, G. P. and Rudraraju, R. 2015. Evaluating student learning using concept maps and markov chains, Expert Systems with Applications, 42(7), 3306-3314.

[14] Chou, Y., Sun, C. and Yen, H. 2012. Evaluating the criteria for human resource for science and technology based on an integrated fuzzy AHP and fuzzy DEMATEL approach, Applied Soft Computing, 12(1), 64-71.

[15] Jeremic, Z. and Jovanovic, J. and Gasevic, D. 2012 Student modelling and assessment in intelligent tutoring of software patterns, Expert Systems with Applications, 39(1), 210-222.

[16] Zadeh, L.A. 1965. Fuzzy Sets, Information and Control. $8,338-353$

[17] Triantaphyllou, E. 2000. Multi-criteria Decision Making Methods: A Comparative Study, Vol. 44, Springer Science and Bussiness Media.

[18] Garvey, P. R. 2008. Analytical Methods for Risk Management: A System Engineering Perspective. CRC Press, Boca Raton, CRC Press, 243-250.

[19] Yong, D. 2006. Plant location selection based on fuzzy TOPSIS, Int. J. Adv. Manuf. Technol, 28 (7), 839-844.

[20] Chou, C. C. 2003. The canonical representation of multiplication operation on triangular fuzzy numbers, Computers \& Mathematics with Applications, 45 (1011), 1601-1610.

[21] Mehregan M. R. et al. 2011. Application of fuzzy analytic hierarchy process in ranking modern education systems, success criteria, Int. J. of e-Education, eBusiness, e-Management and e-Learning, 1(4), 299-304.

[22] Amiri M. P. 2010. Project selection for oil-fields development by using the AHP and fuzzy TOPSIS methods, Expert Systems with Applications, 37(9), 62186224. 